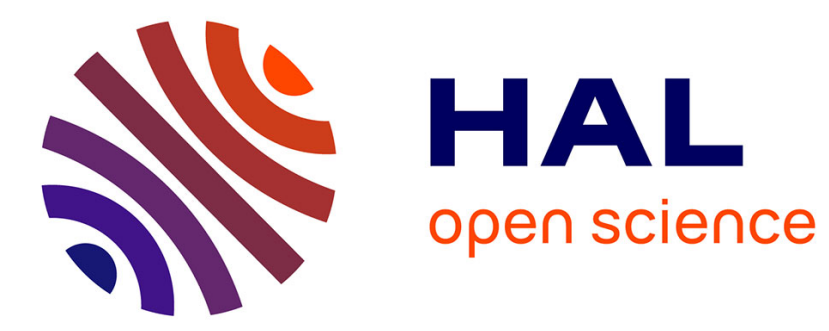

\title{
Probing elastic properties of nanowire-based structures
}

L. Lu, E. Charron, E. Glushkov, N. Glushkova, B. Bonello, F. H Julien, N.

Gogneau, M. Tchernycheva, O. Boyko

\section{To cite this version:}

L. Lu, E. Charron, E. Glushkov, N. Glushkova, B. Bonello, et al.. Probing elastic properties of nanowire-based structures. Applied Physics Letters, 2018, 113 (16), pp.161903 (2018). $10.1063 / 1.5045665$. hal-01913851

\section{HAL Id: hal-01913851 \\ https://hal.sorbonne-universite.fr/hal-01913851}

Submitted on 6 Nov 2018

HAL is a multi-disciplinary open access archive for the deposit and dissemination of scientific research documents, whether they are published or not. The documents may come from teaching and research institutions in France or abroad, or from public or private research centers.
L'archive ouverte pluridisciplinaire HAL, est destinée au dépôt et à la diffusion de documents scientifiques de niveau recherche, publiés ou non, émanant des établissements d'enseignement et de recherche français ou étrangers, des laboratoires publics ou privés. 


\section{PROBING ELASTIC PROPERTIES OF NANOWIRE-BASED STRUCTURES}

L. Lu, ${ }^{1}$ E. Charron, ${ }^{2}$ E. Glushkov, ${ }^{3}$ N. Glushkova, ${ }^{3}$ B. Bonello, ${ }^{2}$ F. H. Julien, ${ }^{1}$

N. Gogneau, ${ }^{1}$ M. Tchernycheva ${ }^{1}$ and O. Boyko ${ }^{2}$

1) Centre de Nanosciences et de Nanotechnologies, UMR 9001 CNRS,

Univ. Paris Sud, Univ. Paris-Saclay, 8 Avenue de la Vauve, 91120 Palaiseau,

France

2) Sorbonne Universités, CNRS, Institut des NanoSciences de Paris (INSP), 75252 Paris cedex 05, France

3) Kuban State University, Institute for Mathematics, Mechanics and Informatics, 350080 Krasnodar, Russia

We report the analysis of elastic properties of a composite medium consisting of GaN nanowires embedded into a dielectric matrix, which constitutes the active material of nanowire-based piezogenerators. A non-invasive technique, Transient Grating Method, is used to generate and analyze the propagation of acoustic waves in the frequency range from a few tens of $\mathrm{MHz}$ to several $\mathrm{GHz}$. The wave propagation is simulated using the time-efficient computer model based on the explicit integral and asymptotic representations for laser-generated surface acoustic waves in layered anisotropic waveguides. Effective parameters of the multilayered model are obtained via the minimization of the discrepancy between the measured and calculated wave characteristics. A comparison with a reference composed of a dielectric matrix without nanowires shows that the mechanical behavior is strongly influenced by the presence of the nanostructures.

Keywords: elastic properties of nanogenerators, GaN nanowires, surface acoustic waves 
Semiconductor nanowires (NWs), i.e., elongated nanocrystals with a sub-micrometer diameter, are today a topic of intense research, motivated by their numerous applications (e.g., light emitting diodes ${ }^{1}$, photodetectors ${ }^{2}$, solar cells ${ }^{3}$, and other energy harvesters ${ }^{4-6}$ ). In particular, these nano-objects can respond to the current need in mechanically flexible devices such as wearable intelligent electronics, bendable light sources, flexible screens, etc ${ }^{7,8}$. Semiconductor nanowires show remarkable mechanical properties ${ }^{9}$ stemming from their small diameter, high aspect-ratio, and defect-free structure. They are indeed mechanically flexible and can stand high deformations without plastic relaxation.

Among different semiconductors, the nanomaterials possessing high piezoelectric coefficients, such as $\mathrm{ZnO}$ and III-nitrides, have attracted special attention for mechanical energy harvesting, force sensing and light emission with a piezoelectric control over the optical signal (so-called piezophototronic effects ${ }^{10}$ ). The piezoelectric coefficients were shown to be enhanced in the nanowires with respect to the bulk counterparts. This size effect was both theoretically predicted ${ }^{11}$ and experimentally demonstrated by atomic force microscopy (AFM) measurements ${ }^{4,12}$ at a single nanowire scale. Thanks to these promising properties, nanowires embedded in a dielectric matrix represent a unique piezoelectric composite medium, which can be used as an active layer in piezoelectric generators for mechanical to electrical energy conversion. For macroscopic piezogenerators based on dielectric-embedded nanowires, a power output of $2.7 \mathrm{~mW} / \mathrm{cm}^{3}$ and $12.7 \mathrm{~mW} / \mathrm{cm}^{3}$ was reported for $\mathrm{ZnO}^{13}$ and $\mathrm{GaN}^{14}$ nanowires, respectively. Despite these encouraging results, there is still a lack of accurate understanding of the deformation and subsequent piezogeneration created inside the 1D-nanostructures in a macroscopic device, which hinders the optimization of the piezogenerator efficiency. One of the main reasons lies in the poor knowledge of the device mechanical characteristics, whereas for all applications requiring mechanical flexibility, and especially for piezogenerators, the knowledge of elastic properties of the nanowire/dielectric composite medium is important.

Although there is a large number of works devoted to nanowire piezogenerators ${ }^{5,13,14}$, there has been much less research addressing the elastic properties of the active layer of these devices. Several investigations of mechanical properties were done on free-standing single nanowires, piezoelectric ${ }^{15,16}$ or not $^{17,18}$. Namely, Young modulus of single GaN nanowires was measured using electromechanical resonance analysis in a transmission electron microscope. In particular, it has been shown that the Young modulus is strongly different in the NW 

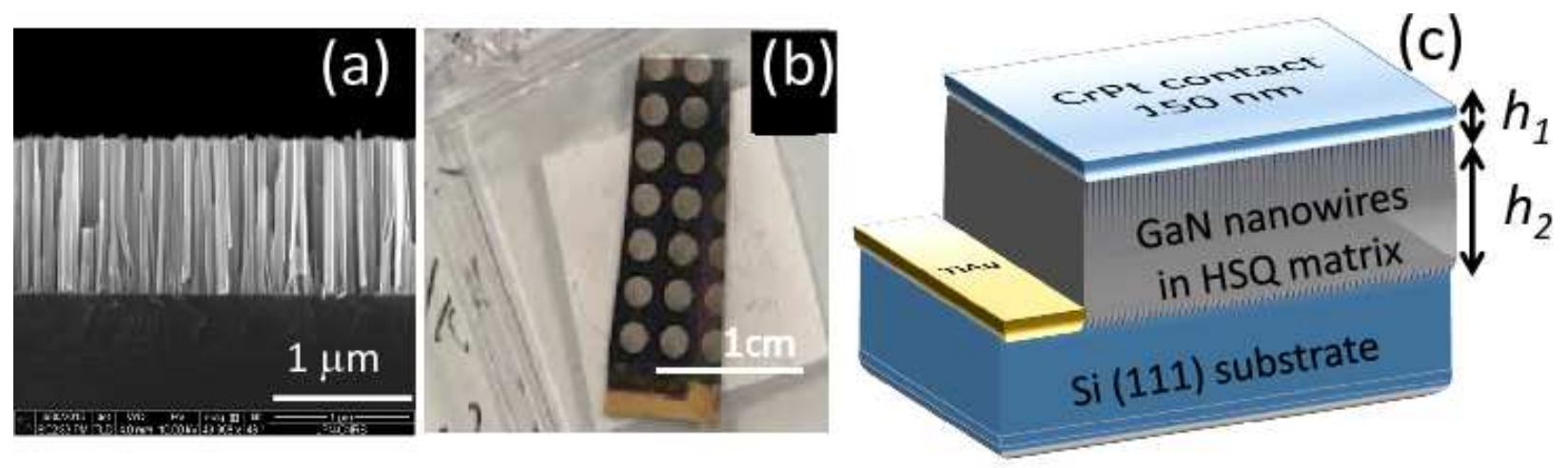

FIG. 1. Scanning electronic microscope picture of GaN nanowires on n-doped Si (111) substrate before the encapsulation into a polymer matrix (a); sample with $\mathrm{Cr} / \mathrm{Pt}$ and $\mathrm{Ti} / \mathrm{Au}$ contacts (b); Samples' structure (c): upper CrPt contact, thickness $h_{1}=150 n m$; bottom contact Ti/Au, GaN nanowires embedded in HSQ matrix layer of thickness $h_{2}=1 \mu m$.

case with respect to the bulk material. However, for the device operation, it is important to consider not only free-standing nanowires but also the surrounding material. The elastic properties of a nanowire array embedded into a dielectric matrix have not been analyzed yet. To quantitatively measure nanoscale deformation of such a device and to analyse precisely this deformation in terms of elastic constants and wave's velocities, we need to develop a appropriated methodology.

Actually, there are not many experimental techniques well suited to measure the mechanical properties such as sound velocity and dispersion in composite systems. When the device includes piezoelectric elements, electromechanical transducers and network analysers can be used for characterization but with the disadvantage of a poor frequency tuning. The non-contact technique based on the Transient Grating Method (TGM) that we use in the present investigation allows for characterizing the elastic properties of almost any kind of materials, transparent or opaque, ordered or disordered, piezoelectric or not in a frequency range that stretches from a few tens of $\mathrm{MHz}$ up to several $\mathrm{GHz}^{19-21}$. The key aspect here is the frequency tuning in the acoustic multi-modal region where dispersion curves have important variations and are strongly sensitive to the layers parameters. This makes it possible to restore effective elastic properties from wave characteristics.

In this work, we use this TGM to explore the guided wave characteristics in a fully functional piezogenerator based on GaN nanowires embedded into a dielectric matrix (hydrogen 
silsesquioxane (HSQ)). The propagation of surface acoustic waves (SAWs) is explored in a frequency range from $100 \mathrm{MHz}$ to $1.4 \mathrm{GHz}$. By detecting the elastic wave propagation at the device surface and analyzing the dispersion curves, both the effective Young modulus and Poisson's ratio of the composite layer can be extracted. The medium is found to be highly anisotropic between the in-plane and out-of-plane directions due to the presence of vertically-aligned NWs. To simulate the SAW characteristics in such waveguide structures, explicit representations for forced-generated guided waves $(\mathrm{GWs})$ have been used ${ }^{22,23}$. Effective elastic moduli of the composite HSQ-NW medium are obtained via the minimization of the discrepancy between the measured and calculated wave characteristics. A comparison with a reference composed of a dielectric matrix without NWs shows that the mechanical behavior is strongly influenced by the presence of the nanostructures.

The sample studied here is a piezogenerator based on GaN nanowires grown by PlasmaAssisted Molecule Beam Epitaxy ${ }^{14}$. GaN nanowires are spontaneously formed under nitrogen-rich growth conditions. Vertical NWs with growth direction aligned with the [000-1] crystallographic axis of GaN are formed on a doped 4 nm-thick AlN buffer layer on an n-doped $\mathrm{Si}(111)$ substrate (thickness $=260 \mu \mathrm{m}$ ). The NW morphology is illustrated in Fig 1 (a). The NWs have an average height of $1 \mu m$ and an average diameter of 50 $\mathrm{nm}$ and a density of $10 \mathrm{NWs} / \mu \mathrm{m}^{2}$. To fabricate a piezogenerator, a HSQ polymer was spin-coated onto the NW array and an annealing step at $400^{\circ} \mathrm{C}$ was performed to polymerization into $\mathrm{SiO}_{x}$. Then reactive ion etching was used to remove excessive polymer from the NW summits for contact deposition. Finally, after a de-oxidation of the NW top part, chromium-platinum ( $\mathrm{Cr} 5 \mathrm{~nm} / \mathrm{Pt} 145 \mathrm{~nm}$ ) contacts were deposited through a shadow mask with $2 \mathrm{~mm}$ openings on the top of the NW arrays and a bottom Ti/Au contact was made on the conducting silicon substrate. Fig. 1 (b) shows the fabricated device and Fig 1 (c) illustrates its structure. Cr thin films are used only as an adhesion layer and its thickness is $5 \mathrm{~nm}$. A reference sample without active NWs was also fabricated. It has the same structure as the piezogenerator, but the HSQ-NW layer is replaced by a pure HSQ layer. For the same spin-coating procedure, the thickness of a bare HSQ layer was smaller (250 $\mathrm{nm})$, which was taken into account in the modeling.

The $\mathrm{TGM}^{19}$ is a technique that consists in exciting the vibrations in the sample by illuminating its surface with two infra-red (IR) light pulses (wavelength=1064 nm; 30 ps in duration) produced by diffraction of the laser beam on diffractive optical element. These IR 




FIG. 2. Optical set-up and laser system for TGM experiment in reflection geometry. Two convergent lens; M: mirror; DOE: diffractive optical element.

light pulses produce interference fringes with a period of $\Lambda$. As a consequence of photoelastic processes, elastic GW with the wavelength $\Lambda$ are excited at certain frequencies $f=\Omega / 2 \pi$ conditioned by the dispersion properties of the laminate waveguide structure of the sample shown in Fig. 1(c). The wavelength $\Lambda$ can be easily tuned by setting the angle between the two incident beams via adjusting of lenses focal lengths. The detection of elastic waves is made using a heterodyne scheme. This involves two continuous laser beams (the probe and the reference beams, wavelength $=532 \mathrm{~nm}$ ) with an optical angular frequency $\omega$ issued from the same diffractive optical element as the IR pulses both incident on the sample. The probe beam gets partially diffracted at Bragg angle upon reflection on the phase gratting created by the IR interferences, giving rise to back reflection at optical angular frequencies $\omega+\Omega$. This beam is further mixed with the specularly reflected reference beam. A signal proportional to cos $\Omega t$ results from the mixing of both first-order diffracted probe beam and specularly reflected reference beam. This signal is recorded with a fast photodiode connected to a broadband oscilloscope and further processed using conventional fast Fourier transform techniques. The experimental setup is illustrated in Fig. 2. As an example, Fig. 3 depicts a typical spectral amplitude of the signal recorded for the HSQ-NW sample when $\Lambda$ is set to $5.85 \mu \mathrm{m}$ (the signal in time domain is shown in the inset). This spectrum features two main peaks at $f=495$ and $f=565 \mathrm{MHz}$, and a smaller one at $f=854 \mathrm{MHz}$ due to a dumped 


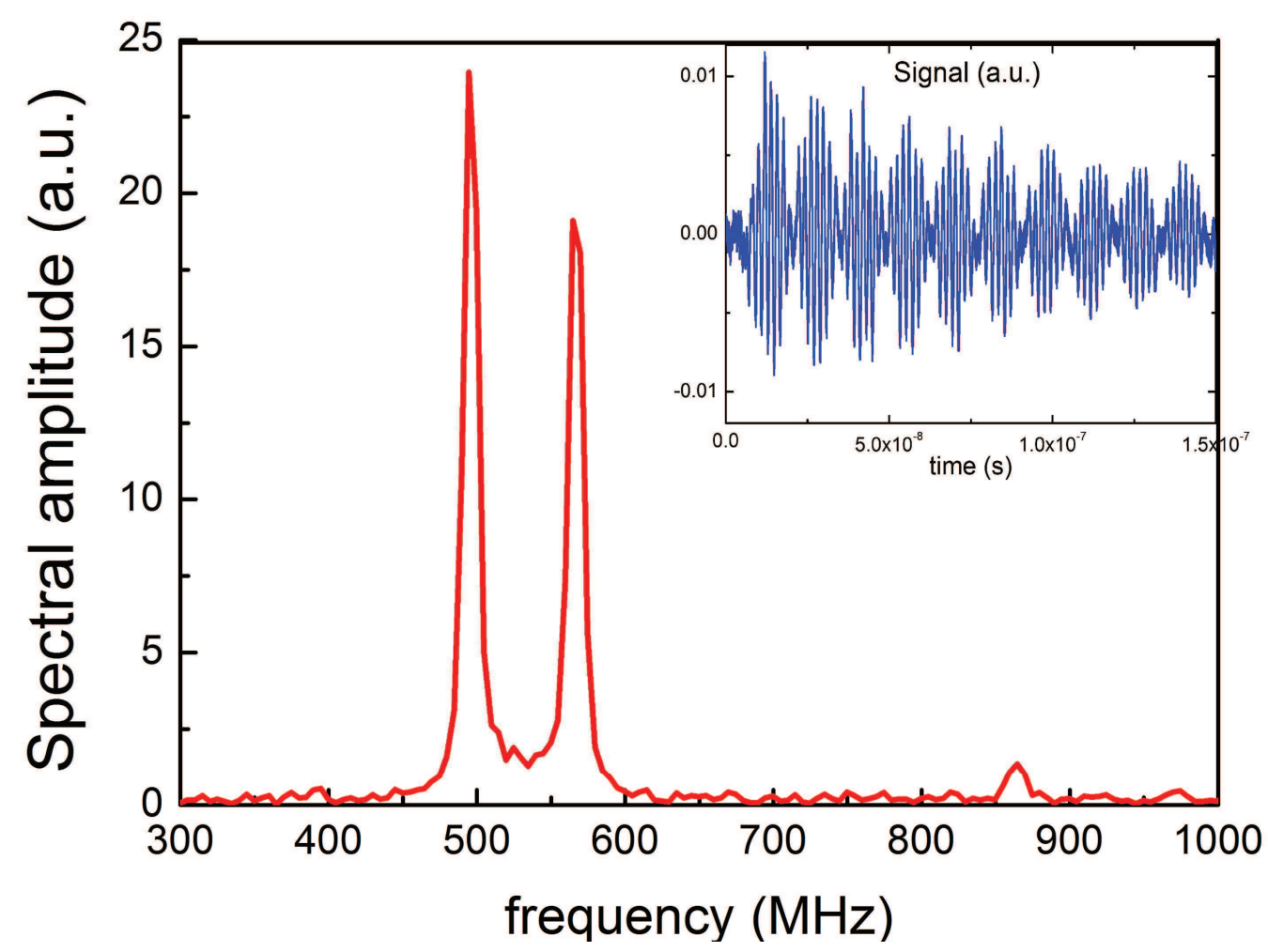

FIG. 3. Frequency spectrum of the laser-generated signal for the HSQ-NW sample shown in the insert; the wavelength of the SAWs is $\Lambda=5.85 \mu \mathrm{m}$.

GW. They correspond to the first SAW modes: the Rayleigh and Sezawa modes.

To simulate the frequency spectra of the generated SAWs, we use the Greens matrix based solution described in Ref. ${ }^{22,23}$. The components of the displacement vector $\mathbf{u}$ obey the elastodynamics equations

$$
C_{i j k l} u_{l, j k}+\rho \Omega^{2} u_{i}=0, \quad i=1,2,3 .
$$

The elastic stiffness tensor $C_{i j k l}$ and the density $\rho$ are piecewise constant functions of the transverse coordinate $z$, thereby keeping constant values within the sublayers of thickness $h_{m}, \quad m=1, \ldots, M$. The bottom substrate is a half-space $\left(h_{M}=\infty\right)$. The outer surface $z=0$ is stress free, except within the loading region $D$ :

$$
\left.\boldsymbol{\tau}\right|_{z=0}=\mathbf{q} \quad(\mathbf{q}(x, y) \equiv 0 \text { for }(x, y) \notin D),
$$

and the sublayers are perfectly bonded with each other. Here $\boldsymbol{\tau}=\left\{\tau_{x z}, \tau_{y z}, \sigma_{z}\right\}$ is a traction vector at a horizontal surface area $z=$ const; $\mathbf{q}$ is a given load simulating the action of the laser beam generating SAWs. 
The solution to this boundary value problem is obtained in terms of the Green's matrix $k(\mathbf{x})$ and the vector of load $\mathbf{q}$ applied to a surface area $D$, or equivalently, via their Fourier symbols $K\left(\alpha_{1}, \alpha_{2}, z\right)=\mathcal{F}_{x y}[k]$ and $\mathbf{Q}\left(\alpha_{1}, \alpha_{2}\right)=\mathcal{F}_{x y}[\mathbf{q}]$ :

$\mathbf{u}(\mathbf{x})=\iint_{D} k(x-\xi, y-\eta, z) \mathbf{q}(\xi, \eta) d \xi d \eta=\frac{1}{(2 \pi)^{2}} \iint_{-\infty}^{\infty} K\left(\alpha_{1}, \alpha_{2}, z\right) \mathbf{Q}\left(\alpha_{1}, \alpha_{2}\right) e^{-i\left(\alpha_{1} x+\alpha_{2} y\right)} d \alpha_{1} \alpha_{2}$

Here $\mathcal{F}_{x y}$ is the Fourier transform with respect to $x$ and $y$ variables; the Fourier parameters $\alpha_{1}$ and $\alpha_{2}$ play the role of wavenumbers for the waves propagating along the $(x, y)$ surface. The residues from the poles $\zeta_{n}$ of the matrix $K$ elements describe traveling SAWs generated by the source load q. The element $K_{33}$ of the matrix $K$ specifies the vertical displacement response of the multilayered substructure on a normal elementary loading. With the laser generation and measurements it is sufficient to use only this element, i.e., in the Fourier symbols, to restrict ourselves to the relation $U_{3}=K_{33} Q_{3}$. For SAW propagating along the $x$ axis $\left(\alpha_{2}=0\right)$, the characteristic equation that relates the wavelength $\Lambda=2 \pi / \alpha_{1}$ with frequency $f$ can be written in the form

$$
K_{33}^{-1}(2 \pi / \Lambda, 0, f)=0 .
$$

With a fixed wavelength $\Lambda$, the roots of this equation $f_{n}$ are the frequencies of SAW modes, which should coincide with the TGM measured frequencies of samples resonance response, e.g., peak frequencies in Fig. 3. With a fixed frequency $f$, the roots yield the SAW wavenumbers $\zeta_{n}=2 \pi / \Lambda_{n}$ and, correspondingly, their phase velocities $c_{n}=\Omega / \zeta_{n}$ or inverse to them slownesses $s_{n}=\zeta_{n} / \Omega$. For independent reproducing of the numerical results below, one can use the algorithm of matrix $K$ calculation for a multilayered elastic structure of arbitrary anisotropy, which is exhaustively described in Ref. ${ }^{22}$.

Accordingly, to obtain the effective moduli of the sample by varying the parameters of the multilayered model to fit the calculated SAW dispersion characteristics to the experimental points, we use the cost function of the following form:

$$
F(C, \rho, h)=\sum_{j}\left|K_{33}^{-1}\left(2 \pi / \Lambda_{j}, 0, f_{j}\right)\right|
$$

The required effective parameters (the matrix of elastic moduli $C$, the density $\rho$ and the thickness $h$ of each sublayer) should minimize this function. To search for min $F$, various approaches can be used, for example, a popular now genetic algorithm. In our case, the 
method of coordinatewise minimization starting from physically expected initial values has proved itself well.

We applied this method to the reference (Fig. 4) and the HSQ-NW (Fig. 5) samples . The colored level-line plot in Fig. 4 (a) depicts a surface plot of $\left|K_{33}\right|$ as a function of the wavelength $\Lambda=2 \pi / \alpha_{1}$ in the $x$-direction $\left(\alpha_{2}=0\right)$ and frequency $f$ for the HSQ reference sample. The areas of $K_{33}$ maximal amplitudes correspond to the parameters of the dominant SAWs. They yield those peaks in the frequency spectra that are detected in the experimental measurements for fixed wavelength $\Lambda$ for both the reference and the HSQ-NW samples (see Fig. 3). In Figures 4 and 5, these experimentally obtained peak points are shown by dots. The ridges of $K_{33}$ amplitude go along the lines specified by its real and nearly real poles $\alpha_{1}=\zeta_{n}(f)$ that determine the dispersion characteristics of the generated traveling SAWs and leaky guided waves; viz., the SAWs phase velocities are $c_{n}=\Omega / \zeta_{n}$ while the inverse to their slownesses are $s_{n}=\zeta_{n} / \Omega$. The latter more conveniently allows analyzing the SAWs dispersion properties, since, in contrast with the phase velocity curves, their magnitudes vary in a limited range specified by the frequency-independent body-wave slownesses shown in Fig. 4 (b) and Fig. 5 by horizontal dashed lines. They are indicated by the symbols $P 1$, $P 2, P 3$, and $S 1, S 2, S 3$, which relate to longitudinal and transversal ( $P$ and $S$ ) bulk waves propagating in the $x$-direction in each of the sublayers numbered from top to bottom.

To obtain the effective moduli of the HSQ interlayer, its material parameters, which are used as an input for the code calculating $K_{33}$, are varied to fit the experimental points. The algorithm is based on the minimization of predetermined cost function. Only the Si layer (thickness and elastic constants) kept constant, Pt and HSQ layers parameters are restored by best fit to the data. The real branches of the SAW slowness curves start from the S3 level ( $S$-waves in the lowest (third) sublayer consisting of $\mathrm{Si}[111])$ and tend to the Rayleigh wave slowness in the half-space with the properties of the upper layer (composed of platinum) as $f \rightarrow \infty$.

Figure 5 presents the same analysis for the sample CrPt/HSQ-NW/Si with GaN NW. The curves in Fig. 5 are for the three-layered structure with the restored material properties of the HSQ-NW composite material and layer thicknesses, $h_{1}=0.150 \mu m$ and $h_{2}=1.059$ $\mu m$. It is clearly seen that the computed fitting curves of Fig. 5 taking into account the NWs approximate well the experimental data points. The parameters providing the best fit of Fig. 4 and Fig. 5 are summarized in Table I. The table displays the restored densities 
a)

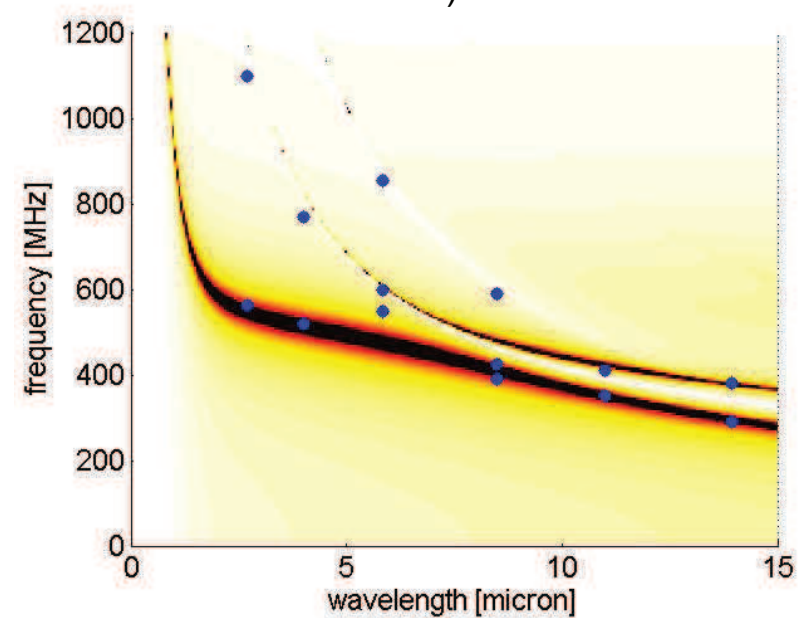

b)

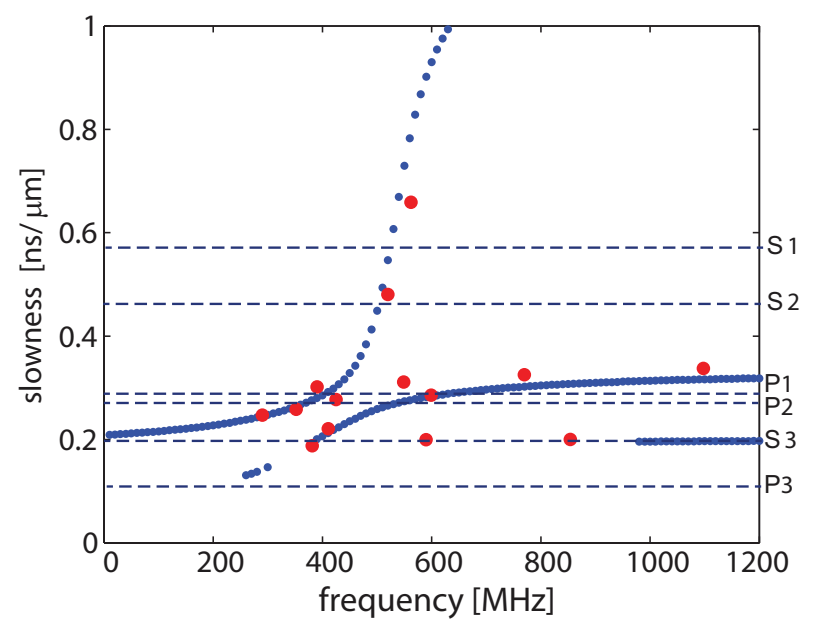

FIG. 4. The level-line plot of the Greens matrix $\left|K_{33}\right|$ element as a function of wavelength $\Lambda$ and frequency $f$ (a) and slowness dispersion curves (b) for the reference sample CrPt/HSQ/Si without nanowires (input and restored material parameters are in Table I ; $h_{1}=0.137 \mu \mathrm{m}$ and $h_{2}=0.250$ $\mu m)$; dots indicate the points of experimentally obtained peaks of SAW frequency spectra at prescribed wavelengths $\Lambda$; dashed horizontal lines are for $P$ and $S$ body-wave slownesses in the sublayers numbered from top to down.

TABLE I. Elastic moduli $C_{i j}[\mathrm{GPa}]$, density $\rho\left[10^{3} \mathrm{~kg} / \mathrm{m}^{3}\right]$, material constants $E_{x y}$, $E_{z}$, and $\mu_{z}$ $[\mathrm{GPa}] ; \nu_{x y}$ and $\nu_{z}[$ non-dimensional $]$.

\begin{tabular}{lcccccccccccc}
\hline \hline & $C_{11}$ & $C_{33}$ & $C_{12}$ & $C_{13}$ & $C_{44}$ & $C_{66}$ & $\rho$ & $E_{x y}$ & $E_{z}$ & $\mu_{z}$ & $\nu_{x y}$ & $\nu_{z}$ \\
\hline PtCr input & 315 & 315 & 193 & 193 & 61.0 & 61.0 & 21.5 & 168 & 168 & 61 & 0.380 & 0.380 \\
Si[111] input & 195 & 205 & 54.3 & 44.7 & 60.7 & 70.3 & 2.33 & 175 & 189 & 60.7 & 0.241 & 0.179 \\
PtCr restored with HSQ & 262 & 262 & 130 & 130 & 65.8 & 65.8 & 21.5 & 175 & 175 & 65.8 & 0.332 & 0.332 \\
HSQ restored & 9.58 & 9.58 & 3.02 & 3.02 & 3.28 & 3.28 & 0.70 & 8.13 & 8.13 & 3.28 & 0.240 & 0.240 \\
HSQ-NW restored & 2.00 & 129 & 0.53 & 1.26 & 7.12 & 0.74 & 2.06 & 1.85 & 128 & 7.12 & 0.260 & 0.500 \\
\hline \hline
\end{tabular}

and the related material constants: $C_{i j}$, Young moduli $E_{x y}$ and $E_{z}$, shear modulus $\mu_{z}$ and Poisson's ratios $\nu_{x y}$ and $\nu_{z}$. As seen from Table I, the Young modulus for HSQ-NW layer is strongly anisotropic: the out-of-plane Young modulus is almost two orders of magnitude larger than its in-plane counterpart.

For piezogenerators, the mechanical properties of the active medium are important to 


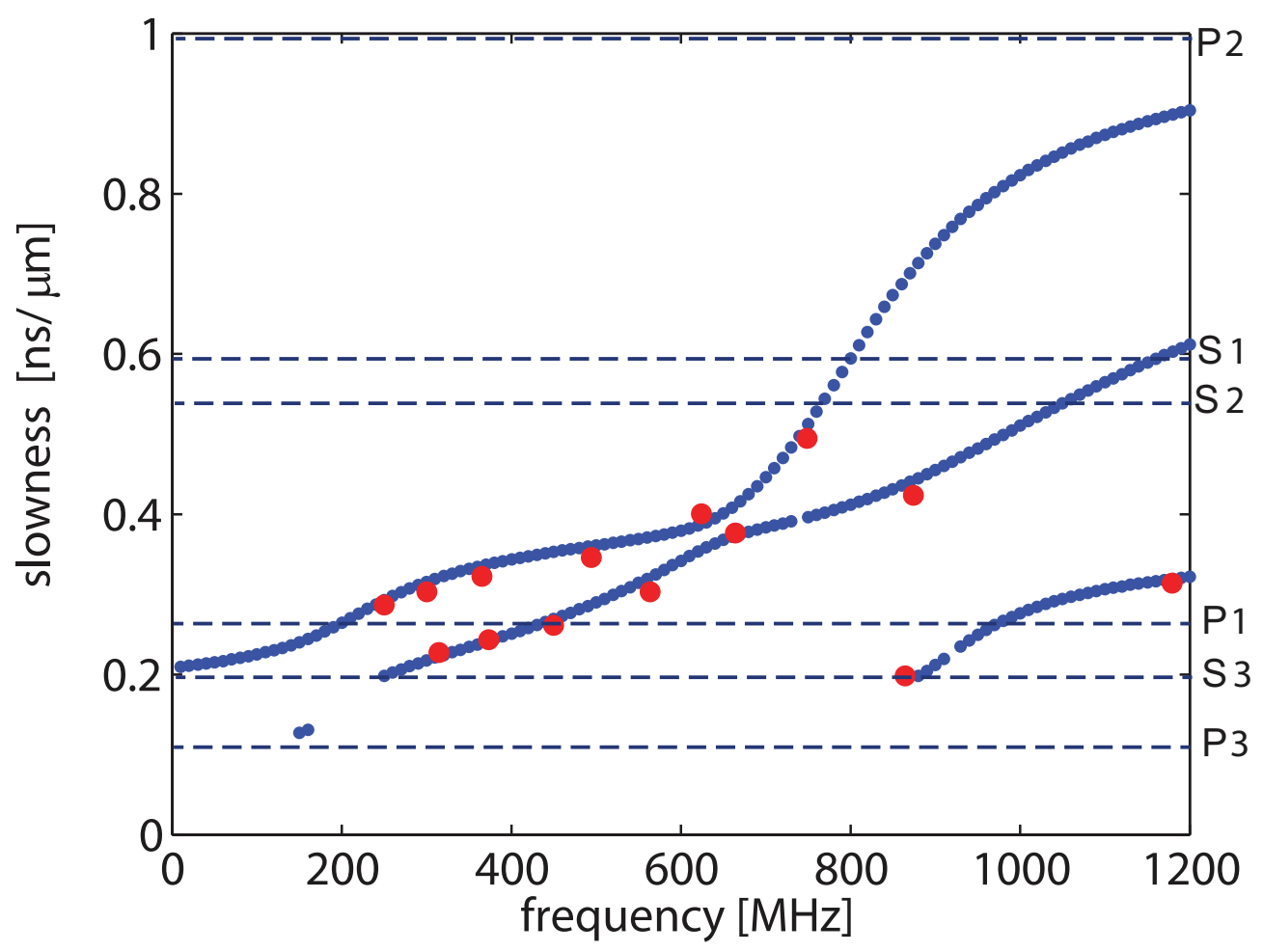

FIG. 5. Experimental data (red dots) for the sample with nanowires CrPt/HSQ-NW/Si and SAW dispersion curves for the structure with restored material constants of HSQ-NW interlayer (Tables I); $h_{1}=0.150 \mu m$ and $h_{2}=1.059 \mu m$.

quantify the response of the device to an external force. In particular, the ability of the generator to respond to small solicitations critically depends on its Young modulus, which should be determined in order to build a reliable model of the piezogenerator. We compare the Young moduli extracted using our TGM method with the experimental data available from the literature for GaN and HSQ. Concerning HSQ, the Young modulus (and $C_{i j}$ ) depends on its degree of polymerization, which is a function of its annealing temperature ${ }^{24}$ and time ${ }^{25}$. Between 350 and 460C, the deformation of the HSQ layer due to the various stresses decreases as the annealing temperature increases, although the stress in the layer due to its polymerization becomes stronger. It has been estimated that in this temperature range, the Young's modulus of the polymer increases from $2 \mathrm{GPa}$ to $7 \mathrm{GPa}^{26}$. If we compare these results with those from table I, in our case the Young modulus is superior. This higher rigidity of the layer may be explained by the variability of the HSQ properties, however the presence of the top metallic electrode in our case may also play a role. Concerning the GaN NWs, it is not easy to benchmark the obtained values, since the values of the Young mod- 
ulus published in the literature are controversial. Indeed, one part of the published studies shows that the Young modulus decreases by reducing the NW diameter ${ }^{16,27}$, while others present the opposite behaviour, i.e. the Young modulus increases with the NW diameter reduction ${ }^{16,28}$. Finally, some publications report the independency of the Young modulus from the wire diameter ${ }^{29}$. If we take a basic isotropic approximation of bulk values for the two constituting materials weighted by the filling factor (33-40\%), the estimated in-plane and out-of-plane Young moduli of our HSQ-GaN NW composite layer are both in the range 103-123 GPa. Comparing these figures with the restored values of table I, we observe that $E_{z} \mathrm{Ez}$ is close to this basic estimation, whereas the $E_{x y}$ is one order of magnitude different. This again evidences the strong mechanical anisotropy of the NW/dielectric composite medium. This again evidences the strong mechanical anisotropy of the NW/dielectric composite medium. Finally, we note that the Young modulus of our GaN NWs/HSQ composite sample is lower than the values reported for free standing GaN NWs, indicating a higher mechanical flexibility of the composite active layer. Since the mechanical flexibility is crucial for a piezogenerator to be able to respond to small external solicitations, this result offers promising prospects for developing high-efficient piezo-generators.

In summary, we have applied the Green's matrix formalism to NW-based nanostructures and we have extracted elastic parameters for the composite NW-dielectric medium. The investigation of dispersion curves leads to the accurate understanding of the elastic properties of embedded NWs and can be further used for the prediction of piezoelectric behavior as well as it can provide a valuable feed-back for the device design optimization. Although the technique has been applied to a specific case of a GaN nanowire-based piezogenerator, the experimental method is not limited to this particular device but can be applied to study elastic properties of other nano-engineered functional materials.

The experimental part of the work was supported by the by EU Horizon 2020 ERC project NanoHarvest (Grant 639052), the French National Research Agency though the GANEX program (ANR-11-LABX-0014), and the theoretical part is supported by the Russian Science Foundation (Project No. 17-11-01191). The authors would like to thank Emmanuel Peronne for helpful discussions. 


\section{REFERENCES}

${ }^{1}$ T. Schimpke, M. Mandl, I. Stoll, B. Pohl-Klein, D. Bichler, F. Zwaschka, J. StrubeKnyrim, B. Huckenbeck, B. Max, M. Müller, P. Veit, F. Bertram, J. Christen, J. Hartmann, A. Waag, H.-J. Lugauer, and M. Strassburg. Physica Status Solidi (a), 213(6):1577-1584, 2016.

${ }^{2}$ C. Soci, A. Zhang, B. Xiang, S. A. Dayeh, D. P. R. Aplin, J. Park, X. Y. Bao, Y. H. Lo, and D. Wang. Nano Letters, 7(4):1003-1009, 2007. PMID: 17358092.

${ }^{3}$ J. Wallentin, N. Anttu, D. Asoli, M. Huffman, I. Åberg, M. H. Magnusson, G. Siefer, P. Fuss-Kailuweit, F. Dimroth, B. Witzigmann, H. Q. Xu, L. Samuelson, K. Deppert, and M. T. Borgström. Science, 339(6123):1057-1060, 2013.

${ }^{4}$ Z. L. Wang and J. Song. Science, 312(5771):242-246, 2006.

${ }^{5}$ Xu S., J. Hansen B., and Lin Wang Z. Nature Communications, 1(93), 2010.

${ }^{6}$ N. Gogneau, N.and Jamond, P. Chrétien, F. Houzé, E. Lefeuvre, and M. Tchernycheva. Semiconductor Science and Technology, 31(10):103002, 2016.

${ }^{7}$ X. Dai, A. Messanvi, H. Zhang, Ch. Durand, J. Eymery, C. Bougerol, F. Julien, and M. Tchernycheva. Flexible light-emitting diodes based on vertical nitride nanowires. Nano Letters, 15(10):6958-6964, 2015. PMID: 26322549.

${ }^{8}$ M.-H. Seo, J.-Y. Yoo, S.-Y. Choi, J.-Sh. Lee, K.-W. Choi, Ch. K. Jeong, K. J. Lee, and J.-B. Yoon. ACS Nano, 11(2):1520-1529, 2017. PMID: 28135071.

${ }^{9}$ H. D. Espinosa, R. A. Bernal, and M. Minary-Jolandan. Advanced Materials, 24(34):46564675 .

${ }^{10}$ Zh. Wang. Piezotronics and Piezo-Phototronics, pages 1-17. Springer Berlin Heidelberg, Berlin, Heidelberg, 2012.

${ }^{11}$ R. Agrawal and H. D. Espinosa. Nano Letters, 11(2):786-790, 2011. PMID: 21241064.

${ }^{12}$ N. Gogneau, P. Chrétien, E. Galopin, S. Guilet, L. Travers, J.-Ch. Harmand, and F. Houzé. Physica Status Solidi (RRL) - Rapid Research Letters, 8(5):414-419, 2014.

${ }^{13}$ S. Xu, Y. Qin, Ch. Xu, Y. Wei, R. Yang, and Z. L. Wang. Nature Nanotechnology, 5:366373, 2010.

${ }^{14}$ N. Jamond, P. Chrétien, F. Houzé, L. Lu, L. Largeau, O. Maugain, L. Travers, J.-C. Harmand, F. Glas, E. Lefeuvre, M. Tchernycheva, and N. Gogneau. Nanotechnology, 27(32):325403, 2016. 
${ }^{15}$ Pierre-Adrien Mante, Sebastian Lehmann, Nicklas Anttu, Kimberly A. Dick, and Arkady Yartsev. Nondestructive complete mechanical characterization of zinc blende and wurtzite gaas nanowires using time-resolved pump-probe spectroscopy. Nano Letters, 16(8):47924798, 2016. PMID: 27352041.

${ }^{16}$ Chang-Yong Nam, Papot Jaroenapibal, Douglas Tham, David E. Luzzi, Stephane Evoy, and John E. Fischer. Nano Letters, 6(2):153-158, 2006. PMID: 16464026.

${ }^{17}$ Cyril Jean, Laurent Belliard, Thomas W. Cornelius, Olivier Thomas, Maria Eugenia Toimil-Molares, Marco Cassinelli, Loïc Becerra, and Bernard Perrin. Direct observation of gigahertz coherent guided acoustic phonons in free-standing single copper nanowires. The Journal of Physical Chemistry Letters, 5(23):4100-4104, 2014. PMID: 26278939.

${ }^{18}$ Yujie Chen, Tim Burgess, Xianghai An, Yiu-Wing Mai, H. Hoe Tan, Jin Zou, Simon P. Ringer, Chennupati Jagadish, and Xiaozhou Liao. Effect of a high density of stacking faults on the young's modulus of gaas nanowires. Nano Letters, 16(3):1911-1916, 2016. PMID: 26885570.

${ }^{19}$ L. Dhar and J. A. Rogers. Applied Physics Letters, 77(9):1402-1404, 2000.

${ }^{20}$ A. A. Maznev. Phys. Rev. B, 78:155323, Oct 2008.

${ }^{21}$ A. Taschin, P. Bartolini, M. Ricci, and R. Torre. Philosophical Magazine, 84(13-16):1471$1479,2004$.

${ }^{22}$ E. Glushkov, N. Glushkova, and A. Eremin. The Journal of the Acoustical Society of America, 129(5):2923-2934, 2011.

${ }^{23}$ E. Glushkov, N. Glushkova, and Ch. Zhang. Journal of Applied Physics, 112(6):064911, 2012.

${ }^{24}$ H.-Ch. Liou and J. Pretzer. Thin Solid Films, 335(1):186 - 191, 1998.

${ }^{25}$ Yvete Toivola, Jeremy Thurn, and Robert F. Cook. Journal of The Electrochemical Society, 149(3):F9-F17, 2002.

${ }^{26}$ Robert F. Cook. Mechanical properties of low dielectric-constant organic-inorganic hybrids. MRS Proceedings, 576:301-312, 1999.

${ }^{27}$ A. Gulans and I. Tale. physica status solidi c, 4(3):1197-1200.

${ }^{28}$ J.J. Brown, A.I. Baca, K.A. Bertness, D.A. Dikin, R.S. Ruoff, and V.M. Bright. Sensors and Actuators A: Physical, 166(2):177 - 186, 2011. 22 nd IEEE International Conference on Micro Electro Mechanical Systems,Sorrento, Italy, 25-29 January 2009.

${ }^{29}$ R. A. Bernal, R. Agrawal, B. Peng, K. A. Bertness, N. A. Sanford, A. V. Davydov, and 
H. D. Espinosa. Nano Letters, 11(2):548-555, 2011. PMID: 21171602. 